\title{
RESEARCH
}

Open Access

\section{Safety and efficacy of dehydrated ethanol soaking of the operative field in the treatment of spontaneous hepatocellular carcinoma rupture}

Jian Sun ${ }^{1,2+}$, Yue Zhu ${ }^{1,3+}$, Yao-rong Peng ${ }^{1,2+}$, Wen-bin Li, ${ }^{1,2}$, He-yun Zhang ${ }^{1,2}$, Zhen-yu Zhou ${ }^{1,2}$, Lin Wang ${ }^{1,4}$, An-de $\mathrm{Ma}^{5}$ and Jie Wang ${ }^{1,2^{*}}$ (i)

\begin{abstract}
Background: The aim of our study was to evaluate the clinical safety and value of ethanol surgical field infiltration (ESFI), combined with distilled water peritoneal lavage (DWPL), after hepatectomy in patients with hepatocellular carcinoma (HCC) rupture.

Methods: Rat liver tissue samples were soaked in dehydrated ethanol for different soaking times, and 18 rats were assigned to three groups that underwent different soaking methods of the hepatectomy cut surface. We retrospectively reviewed 45 patients who underwent hepatectomy for treatment of ruptured HCC. Among these, EFSI combined with DWPL was used in 21 patients (DAW group), with only DWPL used in the other 24 patients (DW group). Clinical outcomes were compared between the two groups.

Results: For in vitro experiments, the depth of coagulation degeneration and necrosis increased with the duration of soaking. For in vivo experiments, rats in all three groups survived until postoperative day 7 without significant postoperative complication. In patients, the rate of post-operation complication was comparable between the two groups $(P=0.398)$, with no between-group differences in liver function levels. The incidence of peritoneal dissemination was significantly higher for DW than DAW group $(P=0.037)$. Kaplan-Meier test identified dehydrated ethanol treatment as a significant factor of disease-free survival (DFS) $(P=0.036)$. On univariate analysis, dehydrated ethanol treatment was associated with better prognostic outcomes, although it was not retained as an independent factor of patient outcome.

Conclusions: Dehydrated ethanol soaking of the cut surface of the hepatectomy could potentially lower the risk of metastasis and improve the effect of hepatectomy for ruptured HCC as well as showed potential therapeutic value for intraoperative iatrogenic rupture of HCC.
\end{abstract}

Keywords: Dehydrated ethanol, Spontaneously rupture, Hepatocellular carcinoma

\footnotetext{
*Correspondence: sumsjw@163.com

${ }^{\dagger}$ Equal contributors

${ }^{1}$ Guangdong Provincial Key Laboratory of Malignant Tumor Epigenetics and Gene Regulation, Medical Research Center, Sun Yat-Sen Memorial Hospital, Sun Yat-Sen University, Guangzhou 510120, China

${ }^{2}$ Department of Hepatobiliary and pancreatic Surgery, Sun Yat-Sen Memorial

Hospital, Sun Yat-Sen University, Guangzhou 510120, China

Full list of author information is available at the end of the article
}

(c) The Author(s). 2018 Open Access This article is distributed under the terms of the Creative Commons Attribution 4.0 International License (http://creativecommons.org/licenses/by/4.0/), which permits unrestricted use, distribution, and reproduction in any medium, provided you give appropriate credit to the original author(s) and the source, provide a link to the Creative Commons license, and indicate if changes were made. The Creative Commons Public Domain Dedication waiver (http://creativecommons.org/publicdomain/zero/1.0/) applies to the data made available in this article, unless otherwise stated. 


\section{Background}

Hepatocellular carcinoma (HCC) is the sixth most prevalent cancer, with an age-adjusted worldwide incidence of 16 cases per 100,000 individuals $[1,2]$. Spontaneous rupture of $\mathrm{HCC}$ is a life-threatening situation that is associated with a poor prognosis, even when treated with surgical resection $[3,4]$. According to the TNM staging system of the AJCC/ UICC (American Joint Committee on Cancer/Union for International Cancer Control), all ruptured HCC tumors are assigned a grade of T4 [5]. Although some studies argue that it is an overestimation to assign a T4 classification to all resectable ruptured tumors, others have demonstrated that spontaneous HCC rupture carries a specific additional negative impact on overall survival (OS) and disease-free survival (DFS) and, therefore, that a 0.5 to 2 stage classification should be added to the baseline tumor stage $[6,7]$.

Curative liver resection is the most effective treatment for ruptured $\mathrm{HCC}$ to improve patient survival. However, this treatment is associated with a high rate of tumor recurrence due to the risk of implanted metastases $[6,8]$. Several methods have been developed in an attempt to decrease the incidence of metastases, including peritoneal lavage with distilled water (DWPL). This conventional technique has been widely used to remove bacteria and tumor cells from the abdominal cavity. However, metastatic tumor cells tend to be agglomerated, rather than existing as individual cells, which makes them difficult to simply be eliminated. Moreover, the cytocidal activity of the DWPL technique is decreased by contamination of the water once it is in contact with the peritoneal cavity, as well as by the limited time window available for lavage to be successful $[9,10]$.

Dehydrated ethanol injected percutaneously (PEIT) has been used for the treatment of nodular-type HCC due to its accessibility and cost-efficacy [11]. In the case of ruptured HCC, PEIT by laparotomy or under ultrasound guidance has been successfully used to stop the bleeding $[12,13]$. Based on this evidence, we hypothesized that dehydrated ethanol could be applied directly on the residual cavity of hepatectomy for ruptured HCC to improve clinical outcomes. Therefore, the aim of our study was to evaluate the safety of using dehydrated ethanol in the operating field in an animal model, as well as to investigate the clinical safety and value of combining ethanol surgical field infiltration (ESFI) with DWPL during hepatectomy performed in patients for the treatment of ruptured HCC.

\section{Methods}

\section{Animals}

Twenty male Wistar albino rats, with a body weight of 200-250 g, were obtained from the Central Animal House of the Sun-Yat Sen University (Guangzhou City, PR China). All animal procedures were conducted according to the guidelines of our institutional animal care center for the ethical use of animals in research, and the protocol was reviewed and cleared by our institution's research ethics committee.

\section{In vitro experiment and histopathological analyses}

Laparotomy was performed in two rats to harvest the left lobe of the liver. The resected liver tissue was cut into $1-\mathrm{cm}$ slices, with tissue slices randomly assigned to the following duration of soaking in a dehydrated ethanol solution: $30 \mathrm{~s}$ and for 1, 3, 5, 10, and $30 \mathrm{~min}$. Slices soaked in saline were used as a control. All liver tissues underwent routine histopathological examination including hematoxylin-eosin (H\&E) and periodic acid-Schiff (PAS) staining. The depth of coagulation necrosis and the extent of fibrous degeneration and infiltration of inflammatory cells were examined in each slice. As well, vessels and bile duct were identified in each slice.

\section{In vivo experiment}

Partial hepatectomy was performed in 18 rats. Serum specimens were collected from the caudal vein preoperatively. To evaluate the effects of ethanol of tissue, animals were randomly assigned to three groups undergoing different conditions of hydropathic compression of the cut hepatectomy surface. In group A, the surface was compressed with an ethanol gauze for $3 \mathrm{~min}$; in group B, with an ethanol gauze for $5 \mathrm{~min}$; and in group $C$, with a saline gauze for $5 \mathrm{~min}$, serving as the control. The ethanol and saline solutions were continuously dripped onto the gauze for the specified duration (Fig. 1a). All rats were sacrificed on postoperative day 7, and 3-ml intracardiac blood samples were drawn followed by examination for intraperitoneal adhesions of the bowel. AST (aspartate transaminase) , ALT (alanine transaminase), ALB (albumin), and TB (total bilirubin) levels were measured from the venous and intracardiac blood samples.

\section{Retrospective case study}

A retrospective cohort study was performed to analyze 45 patients who were treated at our hospital for a ruptured HCC between March 2005 and March 2013: 37 males and 8 females, $44.9 \pm 11.7$ years (range, $22-81$ years), with no history of allergy to alcohol. All patients underwent liver resection, with alcohol soaking performed in the residual cavity of the operative field, followed by DWPL, in 21 patients (DAW group), with the other 24 patients treated only with DWPL as a control group (DW group). Relevant demographic, medical history, and clinical variables were recorded. The severity of liver disease was classified according to the Child-Pugh criteria. Vascular invasion was determined by microscopic and macroscopic infiltration on histopathological examination of tissue samples. Our study protocol involving patients was approved by the Institutional Review Board of Sun Yat-Sen University 

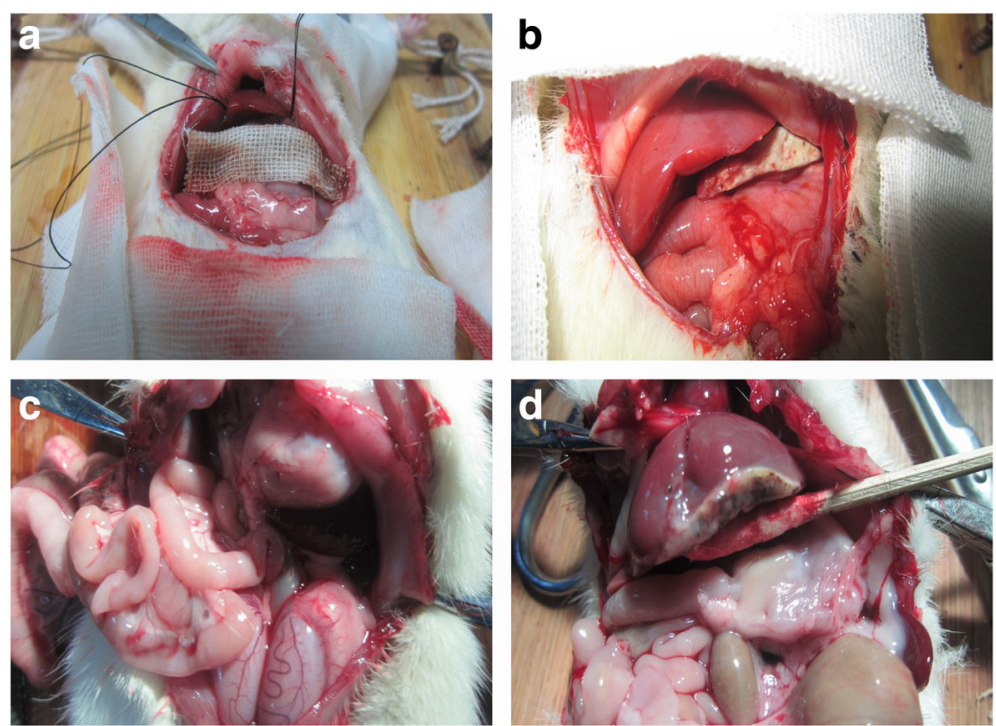

Fig. 1 In vivo experiment. a Partial hepatectomy was performed through a 2.5-cm subcostal incision for 3 groups of rats that underwent different conditions of hydropathic compression of the cut hepatectomy surface. At the time of sacrifice, no severe abdominal adhesions were identified in DW group (b), DAW-3-min group (c), and DAW-5-min group (d)

(Guangzhou, P. R. China), and all patients provided informed consent.

For dehydrated alcohol soaking, the residual cavity of the operative field was surrounded with saline gauze and the cut surface of the hepatectomy was soaked with 50$100 \mathrm{ml}$ of dehydrated alcohol for $5 \mathrm{~min}$. With this process, visible floc becomes visible around the wound and in the surrounding liquid. The anhydrous alcohol was then suctioned off, and the residual anhydrous alcohol film cleaned by a repeated 5-min saline wash until no deposits were visible (Fig. 2a).

For distilled water peritoneal lavage, the residual cavity was washed with 5-6 L of distilled water, divided into at least 3 cycles. In each cycle, the peritoneal cavity was filled with warm $\left(35-40{ }^{\circ} \mathrm{C}\right)$ distilled water and retained for $3 \mathrm{~min}$. The water was then suctioned from the abdominal cavity. The process was repeated until the entire volume of distilled water had been applied, with the entire procedure taking, on average, $15 \mathrm{~min}$.

\section{Statistical analyses}

All data were analyzed using the Statistical Package for Social Sciences (SPSS; version 16.0 for Windows; SPSS Inc., Chicago, IL). Between-group differences were evaluated using Kruskal-Wallis or Mann-Whitney $U$ tests, depending on the normality of the underlying distribution. Data were expressed as a mean \pm SD or median (interquartile range), as appropriate. The significance of clinicopathological features in the 45 patients with ruptured HCC carcinoma was evaluated using chi-squared analysis. Survival rates were calculated using the Kaplan-Meier method, with overall OS defined as the time from hepatectomy until death from any cause or the end of the observation period. DFS was defined at the time from hepatectomy until recurrent disease detection or the end of the observation period without recurrence. Univariate analysis was performed to identify prognostic factors of OS and DFS, with significant variables retained for multivariate analysis and logistic regression. A $P$ value $<0.05$ was deemed to be significant.

\section{Results}

\section{In vitro experiment}

On histological examination, hepatocytes shriveled after soaking in the dehydrated ethanol. Several layers of cells on the surface of tissue samples were spindle-shaped, with decreased cytoplasm that was deeply stained. Nuclear condensation and apoptotic bodies were identified within hepatocytes, with an area of necrosis observable on the most lateral surface of the liver tissue samples. Tissue degeneration, with swelling and pale cells, was identified between the area of necrosis and the inner layers of the tissue samples consisting of normal hepatocytes (Fig. 3b). The depth of coagulation necrosis and degeneration increased as a function of the duration of soaking in dehydrated ethanol, for both tissue samples obtained from the cut hepatectomy surface and the capsule section of the liver (Fig. 3a, c; Table 1).

\section{In vivo experiment}

All rats survived until postoperative day 7 without significant postoperative complications. At the time of sacrifice, no severe abdominal adhesions were identified in any group (Fig. 1b-d). On liver function examination, AST level was significantly higher in group B (5-min ethanol soak) than 

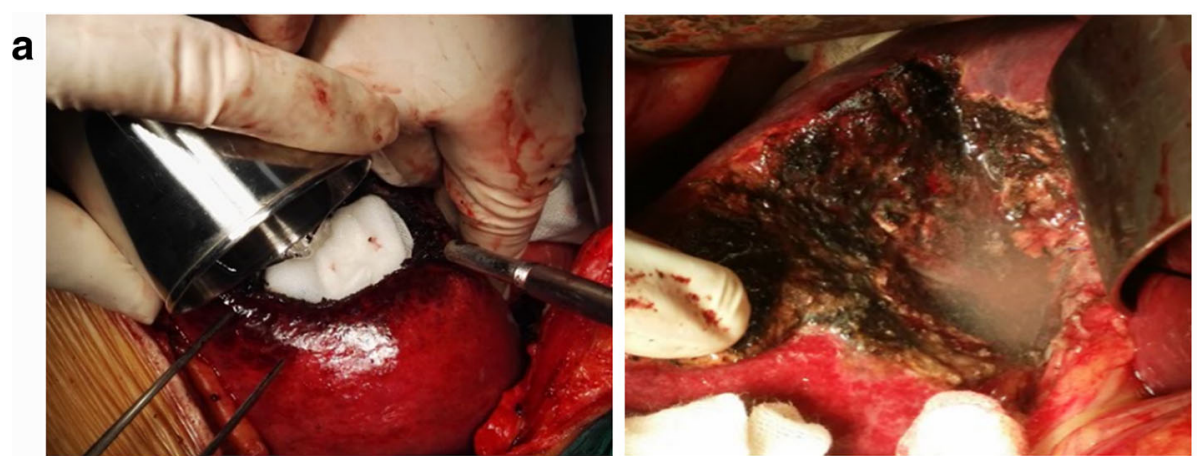

b
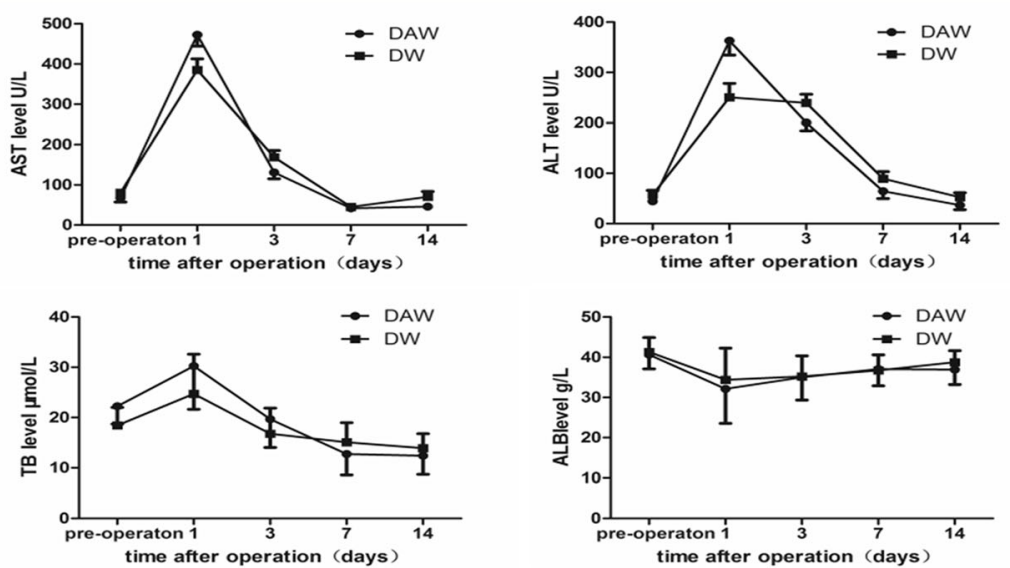

Fig. 2 Retrospective case study. a The cut surface of the hepatectomy was soaked with 50-100 ml of dehydrated alcohol for 5 min; then, the residual anhydrous alcohol films were cleaned by repeated 5-min saline wash until no deposits were visible. $\mathbf{b}$ Levels of AST, ALT, TB, and ALB between the groups at all time points of measurement

that in groups A (3-min ethanol soak; $P=0.028)$ or $\mathrm{C}$ (control; $P=0.017$ ) on postoperative day 3 . ALT level was similarly higher in group $\mathrm{B}$ than in groups $\mathrm{A}(P=0.009)$ or $\mathrm{C}$ $(P=0.014)$ on postoperative day 3. Levels of AST and ALT were comparable between three groups on postoperative day 7. ALB level was also comparable between the three groups on both postoperative days 3 and 7 (Fig. 4).

\section{Retrospective case study}

The clinicopathological features of the 45 patients who underwent curative hepatectomy for a ruptured HCC are summarized in Table 2, with no significant differences between the DAW and DW groups, and all patients surviving the perioperative period. Among the 21 patients in the DAW group, 6 patients developed postoperative complications, including 4 patients with a hydrothorax, 1 with ascites and 1 with a peritoneal infection. Among the 24 patients in the DW group, 5 developed postoperative complications, including 2 patients with a hydrothorax, 2 with ascites, and 1 with a pulmonary embolism. Overall, there was no difference in the incidence rate of postoperative complications between the DAW and DW groups (Table 3; $P=0.398$ ). Levels of AST, ALT, ALB, and TB were comparable between the groups at all time points of measurement: preoperatively and on postoperative days 1 , 3, 7, and 14 (Fig. 2b). Ethanol concentration was evaluated in six patients who underwent hepatectomy followed by ethanol soaking to confirm safety of the dehydrated ethanol procedure after hepatectomy. The maximum blood ethanol concentration identified was $30.1 \mathrm{mg} / \mathrm{dL}$, which is safe for humans (Additional file 1: Table S1).

Follow-up of patients ranged between 1.5 and 125 . 5 months. The OS rate at 1,3 , and 5 years was $71.4,33.3$, and $23.8 \%$, respectively, among patients in the DAW group, compared to 50.0, 25.0, and $12.5 \%$, respectively, among patients in the DW group. The median OS was 24.0 months for the DAW group and 11.6 months for the DW $(P=$ 0.062 ). The DFS rate at 1,3 , and 5 years was $47.6,23.8$, and $14.3 \%$, respectively, among patients in the DAW group, and 29.2, 12.5, and 8.3\%, respectively, among patients in the DW group. The median DFS was 11.5 months in the DAW group, compared to 4.6 months in the DW group $(P=0.006)$. The recurrence rate of $\mathrm{HCC}$ was $76.2 \%$ in the DAW group and $62.5 \%$ in the DW group $(P=0.322)$. The pattern of recurrence was more likely to be extrahepatic or concomitant intrahepatic and extrahepatic, 


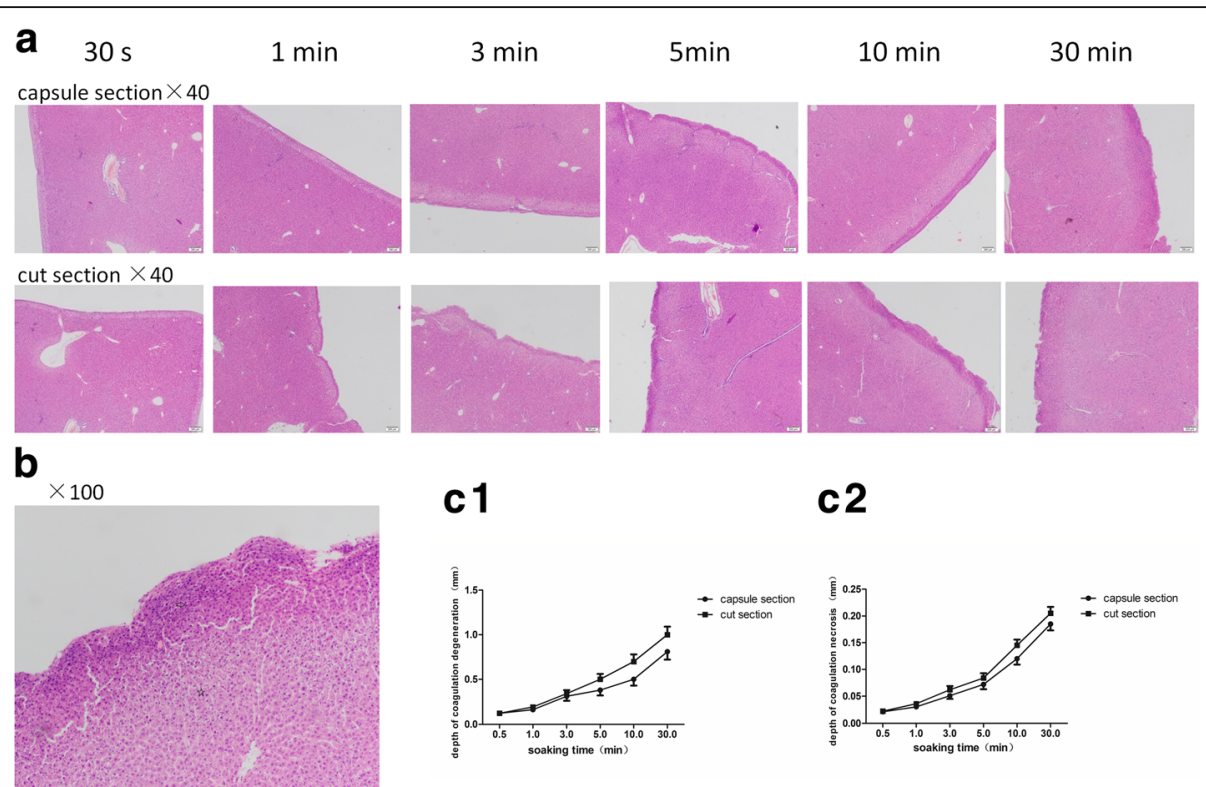

Fig. 3 In vitro experiment. a HE staining of liver tissues for the depth of coagulation necrosis and coagulation degeneration at different soaking times in dehydrated ethanol. $\times 40$ magnification. $\mathbf{b}$ After being soaked in the alcohol, the liver cells emerged to shrivel. Several layer cells of the surface appeared spindle; their cytoplasm was decreased and stained deep (arrow). Some cells appeared to be necrosis, and nuclear condensation or apoptotic bodies in the apoptotic cells were observed with time last. Below the surface layer cells, there were several layers of degeneration cells. These cells had plenty of plasma as the swelling of cells. Therefore, these cells appeared more larger and paler than normal liver cells (star). $\times 100$ magnification. c The depth of coagulation necrosis and degeneration increased as a function of the duration of soaking in dehydrated ethanol, for both tissue samples obtained from the cut hepatectomy surface and the capsule section of the liver

including metastases to the lung, peritoneal cavity, bone, and brain (Table 3). The incidence of peritoneal dissemination was significantly higher in the DW than in the DAW group $(P=0.037)$. The intrahepatic recurrence rate was $47.6 \%$ in the DAW group and $62.5 \%$ in the DW group $(P=0.483)$.

Using the Kaplan-Meier test, type of treatment and other clinicopathologic parameters, such as age, sex, serum AFP level, serum AST level, serum ALT level, Child-Pugh classification, tumor number, tumor size, hepatitis history, tumor differentiation, organ invasion, vascular invasion, and preoperation TACE were not identified as being associated with OS (Fig. 5a). The type of treatment, however, was significantly associated with DFS $(P=0.036$; Fig. 5b). Univariate Cox regression analyses identified better clinical outcomes for patients treated with dehydrated ethanol, although dehydrated ethanol treatment was not identified as an independent predictor of clinical outcome (Table 4).

\section{Discussion}

A ruptured $\mathrm{HCC}$ is considered to be a T4 tumor, according to the TNM staging system, and is associated with a poor clinical prognosis, with tumor cell seeding in the peritoneum increasing the incidence of HCC recurrence [14, 15]. Distilled water lavage during surgery is an established technique to minimize tumor cell seeding after hepatectomy and has been shown to produce positive outcomes $[16,17]$. However, cancer cells can survive the mild hypotonicity of the lavage with distilled water [18]; with the effectiveness of the lavage being further limited by contamination of the water in vivo [9] and the "inoculum size" of exfoliated or

Table 1 The depth of coagulation necrosis and degeneration with the duration of dehydrated ethanol soaking

\begin{tabular}{llllll}
\hline & \multicolumn{2}{l}{ Cut hepatectomy surface } & & \multicolumn{2}{l}{ Capsule section } \\
\cline { 2 - 3 } Time & Coagulation necrosis & Coagulation degeneration & & Coagulation necrosis & Coagulation degeneration \\
\hline $30 \mathrm{~s}$ & $0.022 \pm 0.002$ & $0.12 \pm 0.010$ & $0.021 \pm 0.002$ & $0.12 \pm 0.010$ \\
$1 \mathrm{~min}$ & $0.036 \pm 0.004$ & $0.19 \pm 0.020$ & $0.033 \pm 0.003$ & $0.16 \pm 0.020$ \\
$3 \mathrm{~min}$ & $0.062 \pm 0.007$ & $0.34 \pm 0.040$ & $0.051 \pm 0.006$ & $0.31 \pm 0.050$ \\
$5 \mathrm{~min}$ & $0.084 \pm 0.009$ & $0.50 \pm 0.060$ & $0.072 \pm 0.009$ & $0.38 \pm 0.060$ \\
$10 \mathrm{~min}$ & $0.145 \pm 0.010$ & $1.00 \pm 0.090$ & $0.120 \pm 0.011$ & $0.50 \pm 0.070$ \\
$30 \mathrm{~min}$ & $0.205 \pm 0.012$ & & $0.185 \pm 0.012$ & $0.81 \pm 0.090$ \\
\hline
\end{tabular}

Data were expressed as a mean \pm SD $(\mathrm{mm})$ 


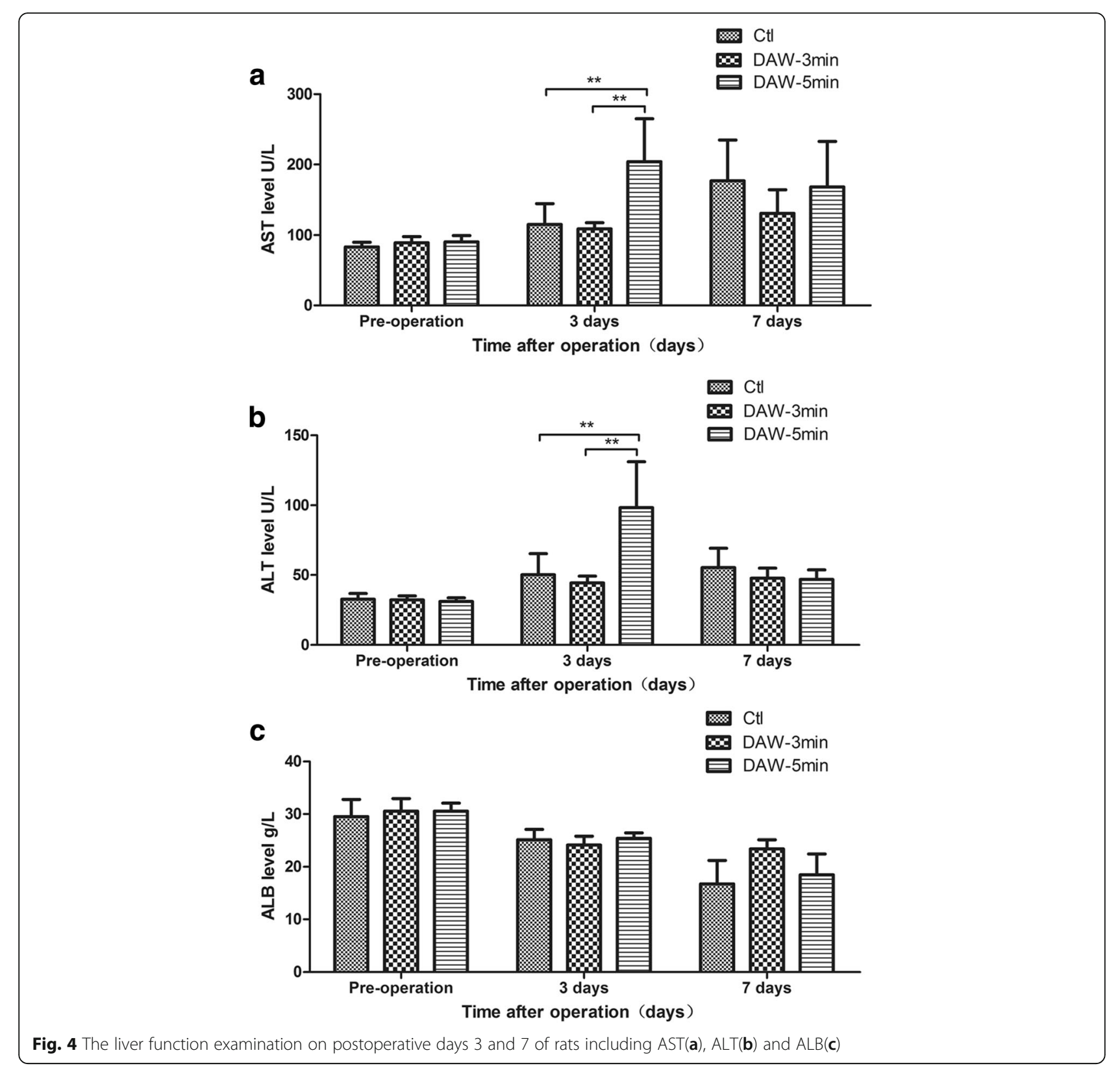

soiled cancer cells [19]. Dehydrated ethanol injection is a widely used technique for the treatment of nodular-type HCC, with demonstrated safety and feasibility. Ethanol induces cellular dehydration, protein denaturation, and chemical occlusion of small tumor vessels, which causes necrosis of HCC lesions [20]. To our knowledge, our study is the first to propose that the use of dehydrated ethanol in the operative field could provide a similar antitumor role as ethanol injected into the liver for treatment of nodular HCC.

We first investigated the relationship between the duration of ethanol immersion and the depth of liver necrosis. In tissue samples of rat liver, the area of necrosis and coagulative degeneration was visible, with a clear boundary.
The depth of necrosis was markedly increased within $10 \mathrm{~min}$, with a maximum depth of $0.2 \mathrm{~mm}$ at the time point of $30 \mathrm{~min}$. The depth of degeneration also markedly increased within $5 \mathrm{~min}$ to a maximum depth of $1 \mathrm{~mm}$. These results are quite different from studies of in vivo ethanol injection into the liver of animals which have reported a large area of necrosis of $1.5 \mathrm{~mm}$, with a spread of ethanol into an area of up to $29 \mathrm{~mm}, 15 \mathrm{~min}$ after injection of $0.3 \mathrm{ml}$ of ethanol [21, 22]. Reasons for these differences include (1) the increase in the diffusion range of anhydrous alcohol by the pressure of the local injection, and (2) blockage of further absorption of the alcohol by the necrotic layer. Therefore, ethanol immersion does not induce severe necrosis and, thereby lowering the risk for 
Table 2 Demographic and clinical characteristics of all patients included in the study

\begin{tabular}{|c|c|c|c|}
\hline & DAW group & DW group & $P$ value \\
\hline & $N=21$ & $N=24$ & \\
\hline Age, years & $48.6(22-81)$ & $46.8(22-71)$ & 0.688 \\
\hline Male to female & $17: 4$ & $20: 4$ & 1.000 \\
\hline HBV positivity ${ }^{a}$ & $16(76.2 \%)$ & $21(87.5 \%)$ & 0.549 \\
\hline Tumor location & & & 0.607 \\
\hline Left lobe & $5(23.8 \%)$ & $6(25 \%)$ & \\
\hline Right lobe & $12(57.1 \%$ & $15(62.5 \%)$ & \\
\hline $\begin{array}{l}\text { Both left and } \\
\text { right lobe }\end{array}$ & $4(19.1 \%)$ & $3(12.5 \%)$ & \\
\hline Child-Pugh classification ${ }^{a}$ & & & 0.551 \\
\hline A & $20(95.2 \%)$ & $22(91.7 \%)$ & \\
\hline B & $1(4.8 \%)$ & $2(8.3 \%)$ & \\
\hline C & 0 & 0 & \\
\hline Pre-operation TACE ${ }^{a}$ & 0 & $3(12.5 \%)$ & 0.236 \\
\hline AST, IU/L & $70.7(29-160)$ & $78.6(22-347)$ & 0.795 \\
\hline ALT, IU/L & $47.8(16-162)$ & $59.3(26-152)$ & 0.507 \\
\hline AFP, IU/mL & $\begin{array}{l}45,705.0 \\
(1.14-225,046)\end{array}$ & $\begin{array}{l}32,254.8 \\
(2.41-129,869)\end{array}$ & 0.662 \\
\hline Tumor size, cm & $9.3(4-20)$ & $8.3(3-21.5)$ & 0.713 \\
\hline Tumor number ${ }^{a}$ & & & 0.127 \\
\hline Single & $16(76.2 \%)$ & $21(87.5 \%)$ & \\
\hline Multiple & $5(23.8 \%)$ & $3(12.5 \%)$ & \\
\hline Vascular invasion ${ }^{a}$ & $12(57.1 \%)$ & $15(62.5 \%)$ & 0.714 \\
\hline Organ invasion ${ }^{\mathrm{a}}$ & $7(33.3 \%)$ & $5(20.8 \%)$ & 0.344 \\
\hline Liver cirrhosis $^{a}$ & & & 0.705 \\
\hline Yes & $3(14.3 \%)$ & $5(20.8 \%)$ & \\
\hline No & 18 (85.7\%) & 19 (79.2\%) & \\
\hline
\end{tabular}

Values in parentheses are medians with range unless indicated otherwise $H B V$ hepatitis B virus, TACE transhepatic arterial chemotherapy and embolization, AST aspartate aminotransferase, ALT alanine transaminase, AFP alpha fetal protein

${ }^{a}$ Values are number with percentages

severe complication, such as bleeding, infection, and leakage.

Secondly, we evaluated the safety of our soaking method using a rat model. Due to the difficulty in creating an operative cavity close to the cut surface for soaking within the small surgical field of the rat liver, we covered the cut surface with gauze and applied a continuous infusion of alcohol to the gauze to ensure a sufficient volume of application of alcohol to the cut surface. Soaking was applied for 3 or $5 \mathrm{~min}$, with the diffusion depth of alcohol increasing quickly up to $5 \mathrm{~min}$. The 3 and $5 \mathrm{~min}$ durations of soaking were selected to limit operative time, which is essential for survival. In our study, all rats survived the procedure without complication. Blood samples confirmed the safety of our ethanol soaking method.
Table 3 Comparison of perioperative and long-term outcomes between DAW and DW group

\begin{tabular}{llll}
\hline & $\begin{array}{l}\text { DAW group } \\
N=21\end{array}$ & $\begin{array}{l}\text { DW group } \\
N=24\end{array}$ & $P$ value \\
\hline Perioperative mortality $^{a}$ & 0 & 0 & N.A. \\
Postoperative complication $^{a}$ & $6(28.6 \%)$ & $5(20.8 \%)$ & 0.398 \\
Recurrence $^{a}$ & & & \\
Intrahepatic $_{\text {Peritoneal dissemination }}$ & $10(47.6 \%)$ & $15(62.5 \%)$ & 0.316 \\
Lung $_{\text {Bone }}$ & $6(28.6 \%)$ & $4(16.7 \%)$ & 0.274 \\
Brain & $2(9.5 \%)$ & $1(4.2 \%)$ & 0.449 \\
\hline
\end{tabular}

Values in parentheses are medians with range unless indicated otherwise

N.A. not applicable

${ }^{a}$ Values are number with percentages

The safety of the procedure was further confirmed by the very low concentration of serum alcohol identified in patients with HCC rupture who underwent ESFI, with no serum alcohol detected in some patients. Reported serum alcohol concentration after alcohol injection into the liver for treatment of nodular HCC was higher than our levels. The lower level of alcohol absorption in our procedure likely reflects the buffering effect of the layer of necrosis. Moreover, our procedure did not produce additional complications or adverse effects, compared to patients who underwent traditional distilled water lavage. Therefore, our method of dehydrated ethanol soaking can be safely used after curative hepatectomy in patients with ruptured HCC.

OS was comparable among patients in the DAW and DW groups, with our rates being comparable to previously reported values. Taku et al. reported 1-, 3-, and 5-year OS rates, after hepatic resection, of 76.0, 48.6, and 33.9\%, respectively [6], with Yang et al. reporting rates of 66.2, 25. 1 , and $16.8 \%$, respectively [3]. However, in our study, ESFI was predictive of a better DFS, with a mean DFS of 11 . 5 months for the DAW group compared to 4.6 months for the DW group. Yang et al. reported DFS rates at 1, 3, and 5 years $40.5,25.8$, and $14.8 \%$, respectively. Jing li et al. reported a median OS of 12 months and DFS of 4 months. On univariate analysis, type of treatment was a prognostic factor only for DFS, with no significant prognostic factor identified for OS. It is important to note that ethanol soaking was not retained as a significant prognostic factor on multivariate analysis. Other known prognostic factors, including the Child-Pugh score, tumor size, and satellite nodule in HCC, were not identified as significant factors, suggesting that ruptured HCC may differ from nonruptured $\mathrm{HCC}$ in terms of biological behavior and pathological characteristics. Differences in liver function and extent of cirrhosis between different study cohorts of patients with ruptured HCC would influence, to some degree, prognostic factors identified on univariate and 

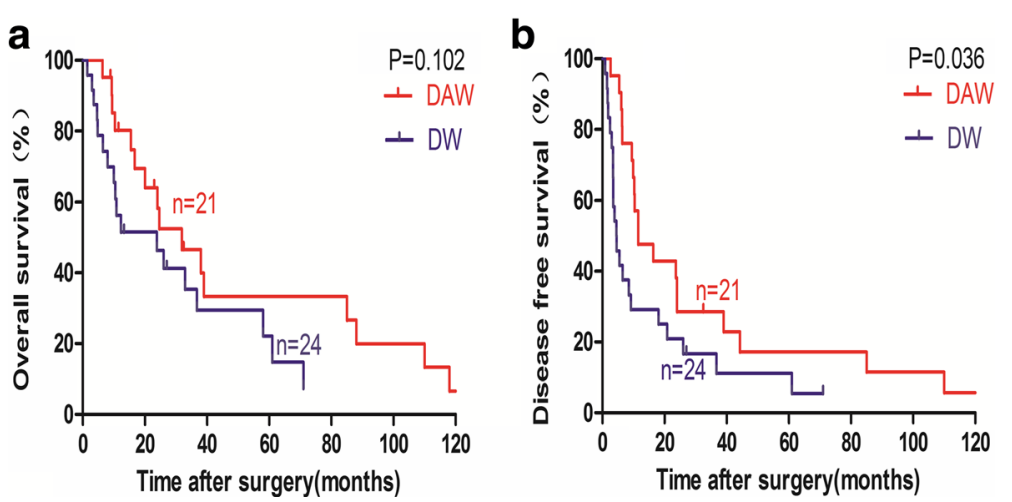

Fig. 5 Kaplan-Meier survival curve of OS (a) and DFS (b) of patients between DAW and DW group. Data for DAW group $(n=21)$ are shown by thick red lines, and data for DW group $(n=24)$ are shown by thick blue line

multivariate analyses. This phenomenon is clearly evident when reviewing previously reported prognostic factors. Shogo et al. reported that the Child-Pugh B was the only independent risk factor of unfavorable OS after hepatic resection for ruptured HCC [23]. A multivariate analysis performed by $\mathrm{Ou}$ et al. identified tumor size, and the number of tumor modules was the only independent factor influencing long-term postoperative survival among patients with ruptured HCC [15]. Jing Li et al. investigated nine variables selected on multivariate analysis to determine the prognostic predictors of survival in patients with spontaneously ruptured HCC, with only radical resection retained as a prognostic factor of OS and DFS [4].

Finally, several limitations of our study warrant mention. Foremost, the clinical data in our study was retrospectively analyzed, and the number of patients with ruptured HCC was relatively small. In addition, although DFS was significantly different between DAW and DW groups, the treatment type failed to be a prognostic factor of postoperative survival.

Table 4 Univariate and multivariate analyses of factors associated with OS and DFS

\begin{tabular}{|c|c|c|c|c|}
\hline \multicolumn{5}{|l|}{ Univariate analysis } \\
\hline \multirow[b]{2}{*}{ Variable } & \multicolumn{2}{|l|}{ Overall survival } & \multicolumn{2}{|l|}{ Disease-free survival } \\
\hline & Hazard ratio $(95 \% \mathrm{Cl})$ & $P$ value & Hazard ratio $(95 \% \mathrm{Cl})$ & $P$ value \\
\hline Age $(\leq 47.0$ vs. $>47.0)$ & $1.045(0.494-2.210)$ & 0.908 & $0.902(0.442-1.840)$ & 0.777 \\
\hline Gender (female vs. male) & $1.618(0.549-4.767)$ & 0.382 & $1.076(0.375-3.089)$ & 0.892 \\
\hline HBsAg (negative vs. positive) & $0.756(0.281-2.029)$ & 0.578 & $1.254(0.481-3.268)$ & 0.643 \\
\hline AFP, IU/mL ( $\leq 400$ vs. $>400)$ & $1.000(0.181-4.645)$ & 0.345 & $1.669(0.443-6.289)$ & 0.276 \\
\hline AST, IU/L ( $\leq 40$ vs. $>40)$ & $1.005(0.996-1.014)$ & 0.257 & $1.004(0.997-1.011)$ & 0.229 \\
\hline ALT, IU/L ( $\leq 40$ vs. $>40)$ & $1.004(0.993-1.015)$ & 0.453 & $1.001(0.990-1.012)$ & 0.864 \\
\hline Child-Pugh classification(A vs. B and C) & $0.458(0.062-3.402)$ & 0.445 & $0.646(0.152-2.732)$ & 0.552 \\
\hline Tumor size, cm ( $\leq 10$ vs.> 10) & $1.062(0.446-2.528)$ & 0.892 & $0.690(0.298-1.598)$ & 0.386 \\
\hline Tumor number (single vs .multiple) & $0.849(0.318-2.267)$ & 0.744 & $0.571(0.218-1.493)$ & 0.253 \\
\hline Liver cirrhosis(no vs. yes) & $0.819(0.326-2.059)$ & 0.671 & $0.605(0.267-1.370)$ & 0.228 \\
\hline Organ invasion(no vs. yes) & $1.158(0.511-2.625)$ & 0.726 & $0.912(0.508-2.298)$ & 0.841 \\
\hline Vascular invasion (no vs. yes) & $1.339(0.535-3.355)$ & 0.533 & $1.074(0.504-2.288)$ & 0.854 \\
\hline Pre-operation TACE (no vs. yes) & $1.228(0.415-3.633)$ & 0.711 & $0.722(0.251-2.073)$ & 0.544 \\
\hline Type of treatment (DAW vs.DW) & $1.828(0.877-3.810)$ & 0.107 & $1.951(1.030-3.693)$ & 0.040 \\
\hline \multicolumn{5}{|l|}{ Multivariate analyses } \\
\hline Type of treatment & N.A. & 0.206 & N.A. & 0.331 \\
\hline
\end{tabular}

Multivariate analysis and Cox proportional hazards regression model were used. Variables were adopted for their prognostic significance by univariate analysis $(P<0.05)$

$\mathrm{Cl}$ confidence interval, N.A. not applicable 


\section{Conclusion}

In summary, for the first time, we presented a novel and safe method to lower the risk of seeding of metastatic cells after liver resection for ruptured HCC and to improve the therapeutic effects of hepatic resection. This method could potentially be used not only for spontaneous rupture of $\mathrm{HCC}$ but also for intraoperative iatrogenic rupture of HCC and even in cases in which tumor cell shedding in a local area is suspected. Further studies are needed to evaluate the effect of these methods in hepatic resection for the ruptured HCC patients.

\section{Additional file}

Additional file 1: Table S1. Blood ethanol concentration of patients after dehydrated ethanol soaked on cut surface of liver during partial hepatectomy. (DOCX $15 \mathrm{~kb}$ )

\section{Abbreviations}

AFP: Alpha fetal protein; ALB: Albumin; ALT: Alanine transaminase; AST: Aspartate aminotransferase; HBV: Hepatitis B virus; HCC: Hepatocellular carcinoma; TACE: Transhepatic arterial chemotherapy and embolization; TB: Total bilirubin

\section{Acknowledgements}

We would like to acknowledge all participating staff, especially Drs. Jun-Yao $\mathrm{Xu}$ and Hai Jiang for their valuable contributions to the study as well as in assisting in the writing.

\section{Availability of data and materials}

The datasets supporting the conclusions of this article are included within the article.

\section{Authors' contributions}

SJ and ZY proposed the study. PYR, LWB, ZHY, and ZZY performed the research and wrote the first draft. WL and MAD collected and analyzed the data. All authors contributed to the design and interpretation of the study and to further drafts. WJ is the guarantor. All authors read and approved the final manuscript.

\section{Ethics approval and consent to participate}

This study was approved by the Ethics Committee of Sun Yat-Sen Memorial Hospital of Sun Yat-Sen University.

\section{Competing interests}

The authors declare that they have no competing interests.

\section{Publisher's Note}

Springer Nature remains neutral with regard to jurisdictional claims in published maps and institutional affiliations.

\footnotetext{
Author details

${ }^{1}$ Guangdong Provincial Key Laboratory of Malignant Tumor Epigenetics and Gene Regulation, Medical Research Center, Sun Yat-Sen Memorial Hospital, Sun Yat-Sen University, Guangzhou 510120, China. ${ }^{2}$ Department of Hepatobiliary and pancreatic Surgery, Sun Yat-Sen Memorial Hospital, Sun Yat-Sen University, Guangzhou 510120, China. ${ }^{3}$ Department of Vascular and Thyroid Surgery, Sun Yat-Sen Memorial Hospital, Sun Yat-Sen University, Guangzhou 510120, China. ${ }^{4}$ Department of Pathology, Sun Yat-Sen Memorial Hospital, Sun Yat-Sen University, Guangzhou 510120, China. ${ }^{5}$ Center for Hygiene Testing and Analysis, Southern Medical University, Guangzhou 510120, China.
}

Received: 7 January 2018 Accepted: 16 April 2018

Published online: 26 April 2018

\section{References}

1. Forner A, Llovet JM, Bruix J. Hepatocellular carcinoma. Lancet. 2012;379: 1245-55. (PMID:24399786).

2. Siegel R, Ma J, Zou Z, Jemal A. Cancer statistics, 2014. CA Cancer J Clin. 2014;64:9-29. (PMID:22353262)

3. Yang T, Sun YF, Zhang J, Lau WY, Lai EC, Lu JH, et al. Partial hepatectomy for ruptured hepatocellular carcinoma. Br J Surg. 2013;100: 1071-9. (PMID: 23754648).

4. Li J, Huang L, Liu CF, Cao J, Yan JJ, Xu F, et al. Risk factors and surgical outcomes for spontaneous rupture of BCLC stages $A$ and $B$ hepatocellular carcinoma: a case-control study. World J Gastroenterol. 2014;20:9121-7. (PMID:25083085).

5. Edge SB, Compton CC. The American Joint Committee on Cancer: the 7th edition of the AJCC cancer staging manual and the future of TNM. Ann Surg Oncol. 2010;17:1471-4. (PMID:20180029).

6. Aoki T, Kokudo N, Matsuyama Y, Izumi N, Ichida T, Kudo M, et al. Prognostic impact of spontaneous tumor rupture in patients with hepatocellular carcinoma: an analysis of 1160 cases from a nationwide survey. Ann Surg. 2014:259:532-42. (PMID:23478524).

7. Chan AC, Dai JW, Chok KS, Cheung TT, Lo CM. Prognostic influence of spontaneous tumor rupture on hepatocellular carcinoma after interval hepatectomy. Surgery. 2016;159:409-17. (PMID:26294087).

8. Miyamoto M, Sudo T, Kuyama T. Spontaneous rupture of hepatocellular carcinoma: a review of 172 Japanese cases. Am J Gastroenterol. 1991; 86:67-71. (PMID:1846058)

9. Huguet EL, Keeling NJ. Distilled water peritoneal lavage after colorectal cancer surgery. Dis Colon Rectum. 2004:47:2114-9. (PMID:15657663).

10. Sweitzer KL, Nathanson SD, Nelson LT, Zachary C. Irrigation does not dislodge or destroy tumor cells adherent to the tumor bed. J Surg Oncol. 1993;53:184-90. (PMID:8331941).

11. Cho YK, Kim JK, Kim MY, Rhim H, Han JK. Systematic review of randomized trials for hepatocellular carcinoma treated with percutaneous ablation therapies. Hepatology. 2009;49:453-9. 19065676.

12. Sunderland GT, Chisholm EM, Lau WY, Chung SC, Leung WT, Li AK. Alcohol injection: a treatment for ruptured hepatocellular carcinoma. Surg Oncol. 1992:1:61-3. (PMID:1341236).

13. Nakaji S, Hirata N, Kobayashi M, Shiratori T, Sanagawa M. Endoscopic ultrasonography-guided ethanol injection as a treatment for ruptured hepatocellular carcinoma in the left hepatic lobe. Endoscopy. 2015;47(Suppl 1):E558-60. (PMID:26610083).

14. Sonoda T, Kanematsu T, Takenaka K, Sugimachi K. Ruptured hepatocellular carcinoma evokes risk of implanted metastases. J Surg Oncol. 1989;41:183-6. (PMID:2545975).

15. Ou D, Yang H, Zeng Z, Luo Y, Yang L. Comparison of the prognostic influence of emergency hepatectomy and staged hepatectomy in patients with ruptured hepatocellular carcinoma. Dig Liver Dis. 2016;48: 934-9. (PMID:27263055).

16. Zhou SJ, Zhang EL, Liang BY, Zhang ZY, Chen XP, Huang ZY. Distilled water lavage during surgery improves long-term outcomes of patients with ruptured hepatocellular carcinoma. J Gastrointest Surg. 2015;19: 1262-70. (PMID:25784370).

17. Chang YM, Hsu KF, Yu JC, Chan DC, Chen CJ, Chen TW, et al. Distilled water peritoneal lavage in patients with rupture hepatocellular carcinoma. Hepato-Gastroenterology. 2013;60:140-3. (PMID:23841121).

18. litaka D, Shiozaki A, Ichikawa D, Kosuga T, Komatsu S, Okamoto K, et al. Blockade of chloride ion transport enhances the cytocidal effect of hypotonic solution in gastric cancer cells. J Surg Res. 2012;176:524-34. (PMID:22261593).

19. Basha G, Ghirardi M, Geboes K, Yap SH, Penninckx F. Limitations of peritoneal lavage with antiseptics in prevention of recurrent colorectal cancer caused by tumor-cell seeding: experimental study in rats. Dis Colon rectum. 2000:43:1713-8. (PMID:11156456)

20. Lencioni R. Loco-regional treatment of hepatocellular carcinoma. Hepatology. 2010;52:762-73. (PMID:20564355).

21. Vehmas T, Taari K, Kivisaari L, Laurila P, Taavitsainen M. Intra- and perihepatic effects of ethanol injections in the pig liver. Scand J Gastroenterol. 1993;28:791-4. (PMID:8235435). 
22. Kairemo KJ, Vehmas T, Bondestam S. A novel method for monitoring liver ethanol injections: activity tissue distribution in rat liver using digital quantitative autoradiography and $\mathrm{CH} 3(14) \mathrm{CH} 2 \mathrm{OH}$. Alcohol. 1999; 19:145-9. 10548158.

23. Tanaka S, Kaibori M, Ueno M, Wada H, Hirokawa F, Nakai T, et al. Surgical outcomes for the ruptured hepatocellular carcinoma: multicenter analysis with a case-controlled study. J Gastrointest Surg. 2016;20:2021-34. (PMID:27718151).

Ready to submit your research? Choose BMC and benefit from:

- fast, convenient online submission

- thorough peer review by experienced researchers in your field

- rapid publication on acceptance

- support for research data, including large and complex data types

- gold Open Access which fosters wider collaboration and increased citations

- maximum visibility for your research: over $100 \mathrm{M}$ website views per year 